\title{
A nonlinear adaptive resilient observer for fouling detection and localization in direct contact membrane distillation systems
}

\author{
Yasmine Marani ${ }^{1,2,+}$, Tania Camelia Touati ${ }^{1,2,+}$, Messaoud Chakir ${ }^{1}$ and Taous Meriem Laleg-Kirati ${ }^{2, *}$
}

\begin{abstract}
Because freshwater source exhaustion is a growing issue, the use of seawater desalination technologies is constantly increasing. More attention is drawn to Direct Contact Membrane Distillation (DCMD), thanks to its high rejection factor and low energy consumption, which makes it a promising sustainable solution for water desalination. Nevertheless, the DCMD system is prone to membrane fouling which alters its produced water's properties, deteriorates the membrane performance, and induces huge operation and maintenance costs if not detected in early stages. Considering the above, the present paper proposes a new approach based on an adaptive resilient observer, not only to detect fouling in DCMD systems but also to localize it with high accuracy to further reduce the maintenance costs. The paper starts by recalling the DCMD model in presence of fouling that was developed using the thermal-electrical analogy and the lumped capacitance method. Then, under Lipschitz conditions of the nonlinear terms, the design of the nonlinear adaptive resilient observer is presented to estimate simultaneously the system's states and the fouling thermal resistance. The proposed observer's convergence proof is given using the Lyapunov method, from which a set of linear matrix inequalities (LMI) is derived in order to obtain the observer's gain. To demonstrate the effectiveness of the proposed method compared to the classical adaptive observer-based method, the states and parameter estimation are compared through numerical simulations under both observer designs. Simulation results reveal that the nonlinear adaptive resilient observer outperforms the adaptive observer.
\end{abstract}

\section{INTRODUCTION}

While the earth's surface is mostly covered with water (i.e. $1.4 \times 10^{9} \mathrm{~km}^{3}$ ), only $2.5 \%$ of it is freshwater, and barely $0.5 \%$ of that amount is easily accessible [1]. According to [1], by 2040, the world's freshwater demand is likely to exceed the available amount. Since seawater is a theoretically inexhaustible resource, seawater desalination turns out to be a very high potential solution to overcome this freshwater scarcity issue.

The high rejection factors of solutes present in water, approximating $100 \%$ [2], makes membrane distillation (MD) a high potential desalination technique. MD is an emerging sustainable desalination method, combining both thermal and membrane-based separation techniques. In this process, thermal energy is used for phase change of the

\footnotetext{
+ Both first author.

* Corresponding author.

${ }^{1}$ Control System Engineering Department, Ecole Nationale Polytechnique, Algiers, Algeria. yasmine.maranilg.enp.edu.dz tania_camelia.touati@g.enp.edu.dz messaoud.chakir@g.enp.edu.dz

2 Computer, Electrical and Mathematical Sciences and Engineering Division (CEMSE), King Abdullah University of Science and Technology (KAUST), Thuwal 23955-6900, Saudi Arabia. taousmeriem.laleg@kaust.edu.sa
}

feed water while a hydrophobic membrane is used to isolate the vapor of distilled water from the feed aqueous solution. The distilled water vapor passes through the membrane from the hot feed side to the cooler permeate side due to the transmembrane pressure. Unlike other desalination processes, MD runs at a lower hydrostatic pressure and lower temperatures [2]. Therefore, it requires much less energy, which makes it a promising sustainable solution for water desalination.

There are several configurations of MD, of which the four main ones are Direct Contact Membrane Distillation (DCMD), Air Gap Membrane Distillation (AGMD), Sweeping Gas Membrane Distillation (SGMD), and Vacuum Membrane Distillation (VMD). These systems share the same feed side layout but differ according to the mechanism used to condense the water vapor [3].

Membrane distillation processes suffer from membrane fouling, which is the aggregation of diverse substances on the membrane surface or within its pores [4]. They can be either organic, inorganic, or biological. Fouling is considered as one of the obstacles facing the the widespread industrial use of membrane technologies [5][6][7]. Not only it deteriorates the membrane performance, but also affects the produced water quality and induces important operation and maintenance costs if not detected in early stages.

The literature proposes several methods for fouling detection in membrane-based processes. Some of them discuss a straightforward approach to detect fouling by incorporating sensors in MD systems [8][9]. Since the use of sensors can be challenging and expensive, monitoring fouling evolution using mathematical tools is more suitable for this application. The most common approach is based on statistical methods [10][11], where fouling is estimated online in membrane bioreactors (MBR) using the derivative of the transmembrane pressure (TMP) per cycle. Some papers discuss other mathematical methods based on observers design, where the fault occurrence is concluded from the estimated profiles like the work presented in [12] using a fuzzy polynomial observer to detect fouling in heat exchangers.

For the particular case of DCMD systems, a nonlinear adaptive observer has been used in [13] to estimate the evolution of fouling by estimating the fouling thermal resistance $R_{s c}$. The authors expressed sufficient conditions in the form of linear matrix inequalities (LMI) to ensure the asymptotic convergence of the state and parameter estimation errors. Nevertheless, for large Lipschitz constants, the LMIs may become non-solvable. Moreover, after a thorough 
investigation of the later observer's performance, some limitations have been observed, especially with regard to fouling localization. The above reasons motivated us to propose a new approach based on the nonlinear adaptive resilient observer developed in [14], which is a robust version of the classical adaptive observer.

An observer is defined as non-resilient if the estimation error diverges for small disturbances on the observer's gain [15]. The actual observer's gain may slightly deviate from its original value obtained from offline calculations. For instance, to compute the adaptive observer's gain, the fouling thermal resistance is assumed to be piece wise constant (its derivative is equal to zero), which doesn't reflect its true nature, as it slowly varies. Thus, its derivative induces a small bias. Therefore, the observer must be able to tolerate certain disturbances and uncertainties on its coefficients. Furthermore, the adaptive resilient observer allows more flexibility for LMI feasibility in a larger space of Lipschitz constants compared to the classical adaptive observer [15].

In this paper, a nonlinear adaptive resilient observer is proposed to estimate and localize fouling in a DCMD system under the assumption that the nonlinear terms are Lipschitz. The observer's gain is computed offline by solving a set of LMIs that guarantees sufficient conditions for the estimated states to converge asymptotically to their real values, while the parameter convergence is ensured by the satisfaction of the persistent excitation condition. Moreover, the DCMD module being divided into $\mathrm{N}$ coupled control-volume cells along the membrane, fouling localization is made possible through estimating a vector of $\mathrm{N}$ fouling resistances $R_{s c}$, one for each cell.

The present paper is structured as follows: Section. II recalls the DCMD model in presence of fouling developed in [13]. In Section. III, the proposed nonlinear adaptive resilient observer design is presented for the specific case of membrane-fouling estimation in DCMD systems along with its convergence proof. To demonstrate the effectiveness of the proposed method versus the classical adaptive observer introduced in [13], the states and parameter estimation are compared through numerical simulations in Section. IV. Finally, concluding remarks are given in Section. V.

\section{DCMD MODEL IN PRESENCE OF FOULING}

Depending on the way depositing particles block the membrane, four membrane fouling mechanisms are distinguished as illustrated in Fig. 1: complete pore blocking, standard pore blocking, intermediate pore blocking and cake filtration. This work focuses only on the case of cake filtration represented by a deposited layer on the surface of the membrane, as illustrated in Fig. 2. The cake filtration, translating into fouling, acts like an additive thermal resistance to the heat transfer mechanism between the bulk feed stream and the membrane. This produces an inferior temperature at the feed-membrane interface and thus a lower partial vapor pressure, reducing at last the
TABLE I

NOMENCLATURE

\begin{tabular}{lll} 
Symbol & Description & Unit \\
\hline$A_{m}$ & Differential cell membrane area & $\mathrm{m}^{2}$ \\
$B_{m}$ & Membrane transport coefficient & $\mathrm{Kg} / \mathrm{m}^{2} \mathrm{sPa}$ \\
$C$ & Thermal capacitance & $\mathrm{J} /{ }^{\circ} \mathrm{C}$ \\
$c_{p}$ & Specific heat of water & $\mathrm{J} / \mathrm{Kg}^{\circ} \mathrm{C}$ \\
$e_{1}$ & State estimation error & \\
$e_{2}$ & Parameter estimation error & \\
$G$ & Production rate & $\mathrm{kg} / \mathrm{h}$ \\
$H_{v}$ & latent heat of vaporization & $\mathrm{J} / \mathrm{Kg}$ \\
$J$ & Mass flux & $\mathrm{Kg} / \mathrm{m}^{2} \mathrm{~s}$ \\
$L$ & Thermal coupling inductor & \\
$N$ & Total number of cells in the DCMD & \\
$Q$ & Heat transfer rate & $\mathrm{W}$ \\
$R$ & Thermal resistance & ${ }^{\circ} \mathrm{C}$ or K \\
$R_{s c}$ & Fouling thermal Resistance & ${ }^{\circ} \mathrm{C} / \mathrm{W}$ \\
$T$ & Temperature & ${ }^{\circ} \mathrm{C}$ or K \\
$x$ & State vector & \\
Subscripts & & \\
$b$ & Bulk & \\
$f$ & Feed & \\
in & Inlet & \\
$m$ & membrane interface & \\
$n$ & Index for cell number & \\
out & Outlet & \\
$p$ & Permeate & \\
term & Terminal cell & \\
$z$ & coupling & \\
\hline
\end{tabular}

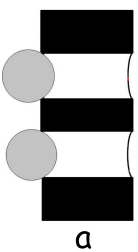

a

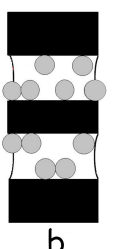

b

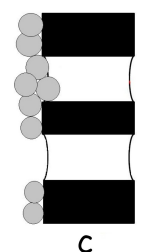

C

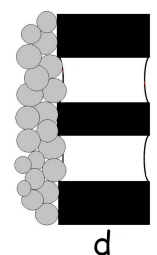

d
Fig. 1. Schema of the fouling mechanisms: (a) complete blocking, (b) standard blocking, (c) intermediate blocking, and (d) cake filtration.

system's production rate.

In order to describe the DCMD system in the presence of fouling, the reduced-order dynamic model derived in [17] is used. The model has been later extended in [13] to take into account the thermal resistance $R_{s c}$. The resulting model has the form of a system of nonlinear differential algebraic equations (DAEs) expressed as follows:

$$
\begin{gathered}
E \dot{x}=A x+B u+D_{1} \gamma(t, x) B_{m}+H\left(x, R_{s c}\right), \\
y=\left[\begin{array}{c}
C x \\
G(x)
\end{array}\right]=\left[\begin{array}{c}
T_{f_{\text {out }}} \\
T_{p_{\text {out }}} \\
\sum_{n=1}^{N} J_{n}(x) A_{m}
\end{array}\right] .
\end{gathered}
$$

Where $x \in \mathbb{R}^{(6 \mathrm{~N}+4) \times 1}$ is the state vector given by: $x=\left[Q_{f_{1}}, T_{b f_{1}}, \ldots, T_{b f_{N}}, Q_{f_{N+1}}, Q_{p_{1}}, T_{b p_{1}}, \ldots, T_{b p_{N}}, Q_{p_{N+1}}\right.$

$$
\left.T_{f_{\text {out }}}, T_{p_{\text {out }}}, T_{m f_{1}}, \ldots, T_{m f_{N}}, T_{m p_{1}}, \ldots, T_{m p_{N}}\right]^{T},
$$

$E$ is a singular matrix whose rank is smaller than $6 N+4$, $A$ is the transition matrix , $u$ is the system's input, i.e. inlet temperatures in the feed and the permeate. $D_{1}$ is a constant matrix linking the nonlinear terms to the state equations. $\gamma(t, x)$ is the nonlinear part of the model. $H\left(x, R_{s c}\right)$ is a nonlinear function of the states $x$ and the fouling thermal 


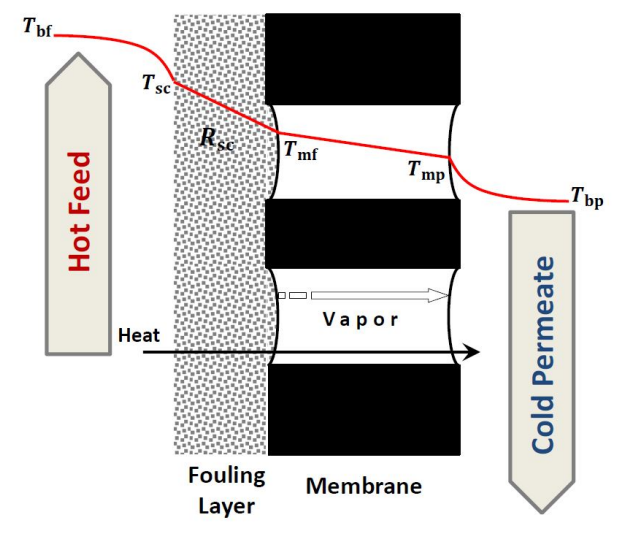

Fig. 2. Schema of the fouling layer in DCMD [13]

resistance $R_{s c}$. $C$ is the output matrix. The production rate $G(x)$ is a nonlinear function which is directly measured at the module's output. The different matrices and functions of (1) are detailed in [17] and their dimensions are given in TABLE II.

TABLE II

\section{MATRICES DIMENSIONS}

\begin{tabular}{|l|l|l|l|}
\hline Matrix & Dimension & Matrix & Dimension \\
\hline$E$ & $(6 N+4) \times(6 N+4)$ & $D_{1}$ & $(6 N+4) \times 3$ \\
\hline$A$ & $(6 N+4) \times(6 N+4)$ & $\gamma(t, x)$ & $3 N \times 1$ \\
\hline$B$ & $(6 N+4) \times 2$ & $B_{m}$ & 1 \\
\hline$C$ & $2 \times(6 N+4)$ & $H\left(x, R_{s c}\right)$ & $(6 N+4) \times 1$ \\
\hline$u$ & $2 \times 1$ & $y$ & $3 \times 1$ \\
\hline
\end{tabular}

The above state space representation is obtained from the equations below, where $n$ refers to the $n^{\text {th }}$ cell, by assuming constant feed and permeate velocities:

$$
\begin{gathered}
\frac{d Q_{f_{n}}}{d t}=\frac{1}{L_{f}^{n}} T_{b f_{n-1}}-\frac{R_{f z}^{n}}{L_{f}^{n}} Q_{f_{n}}-\frac{1}{L_{f}^{n}} T_{b f_{n}} \\
\frac{d T_{\mathrm{bf}_{\mathrm{n}}}}{d t}=\frac{1}{C_{b f}} Q_{\mathrm{f}_{\mathrm{n}}}-\frac{1}{C_{b f}} Q_{\mathrm{f}_{\mathrm{n}+1}}-\frac{1}{C_{b f}}\left(\frac{1}{R_{f}}+J_{n} A_{m} c_{p}\right) T_{\mathrm{bf}_{\mathrm{n}}} \\
+\frac{1}{C_{b f} R_{f}} \frac{R_{s c}\left(1+R_{f} J_{n} A_{m} c_{p}\right)}{R_{f}+R_{s c}\left(1+R_{f} J_{n} A_{m} c_{p}\right)} T_{b f_{n}} \\
+\frac{1}{C_{b f}\left(R_{f}+R_{s c}\left(1+R_{f} J_{n} A_{m} c_{p}\right)\right)} T_{\mathrm{mf}_{\mathrm{n}}} \\
\frac{d Q_{p_{n}}}{d t}=\frac{1}{L_{p}^{n}} T_{b p_{n-1}}-\frac{R_{p z}^{n}}{L_{p}^{n}} Q_{p_{n}}-\frac{1}{L_{p}^{n}} T_{b p_{n}} \\
\frac{d T_{b p_{n}}}{d t}=\frac{1}{C_{b p}} Q_{p_{n}}-\frac{1}{C_{b p} R_{p}} T_{b p_{n}}-\frac{1}{C_{b p}} Q_{p_{n+1}} \\
+\frac{1}{C_{b p}}\left(\frac{1}{R_{p}}+J_{n} A_{m} c_{p}\right) T_{\mathrm{mp}}
\end{gathered}
$$

$$
\begin{aligned}
0= & \left(\frac{1}{R_{f}}+J_{n} A_{m} c_{p}-\frac{1}{R_{f}} \frac{R_{s c}\left(1+R_{f} J_{\mathrm{n}} A_{m} c_{p}\right)}{R_{f}+R_{s c}\left(1+R_{f} J_{n} A_{m} c_{p}\right)}\right) T_{\mathrm{bf}_{\mathrm{n}}} \\
& -\frac{1}{\left(R_{f}+R_{s c}\left(1+R_{f} J_{n} A_{m} c_{p}\right)\right)} T_{\mathrm{mf}_{\mathrm{n}}}-\left(\frac{1}{R_{p}}+J_{\mathrm{n}} A_{m} c_{p}\right) T_{\mathrm{mp}_{n}} \\
& +\frac{1}{R_{p}} T_{\mathrm{bp}_{\mathrm{n}}}, \\
0= & \left(\frac{1}{R_{m}}+\frac{1}{R_{p}}+J_{n} A_{m} c_{p}\right) T_{m p_{n}}-\frac{1}{R_{p}} T_{b p_{n}}-J_{n} A_{m} H_{v}\left[T_{m f_{n}}\right] \\
& -\frac{1}{R_{m}} T_{m f_{n}},
\end{aligned}
$$

$$
\begin{gathered}
0=T_{f_{\text {out }}}-T_{p_{\text {in }}}-R_{\mathrm{f}_{\text {term }}} Q_{f_{N+1}}, \\
0=T_{\mathrm{p}_{\text {out }}}-T_{\mathrm{f}_{\text {in }}}+R_{\mathrm{p}_{\text {term }}} Q_{\mathrm{p}_{1}} .
\end{gathered}
$$

Note that $R_{s c}=0$ represents the ideal case without membrane fouling. Furthermore, as the fouling layer thickens with time, the thermal resistance $R_{s c}$ increases, and the production rate decreases.

It is worth mentioning that the proposed model provides the much needed physical insight into the process while being computationally suitable for real time applications. Moreover, it can be adapted for other MD configurations.

\section{MEMBRANE FOULING DETECTION AND LOCALIZATION}

Expanding the function $H\left(x, R_{s c}\right)$ around $R_{s c}=0$ and considering the linear terms, the following system is obtained:

$$
\begin{aligned}
E \dot{x} & =A x+B u+D_{1} \gamma(t, x) B_{m}+\tilde{H}(x) R_{s c}, \\
y & =\left[\begin{array}{c}
C x \\
G(x)
\end{array}\right] .
\end{aligned}
$$

Where $\tilde{H}(x)$ is the jacobian of $H\left(x, R_{s c}\right)$.

Consider now the nonlinear adaptive resilient observer [14]:

$$
\begin{aligned}
E \dot{\hat{x}} & =A \hat{x}+B u+D_{1} \gamma(t, \hat{x}) B_{m}+\tilde{H}(\hat{x}) \hat{R}_{s c}+[K+\Delta(t)](C x-C \hat{x}), \\
\hat{y} & =\left[\begin{array}{c}
C \hat{x} \\
G(\hat{x})
\end{array}\right] .
\end{aligned}
$$

where $\hat{x} \in \mathbb{R}^{6 N+4 \times 1}$ is the system state estimate and $\hat{R}_{s c} \in \mathbb{R}^{N \times 1}$ the fouling thermal resistance estimate. $K \in \mathbb{R}^{6 N+4 \times 2}$ is the observer gain and the resilient term $\Delta(t) \in \mathbb{R}^{6 N+4 \times 2}$ is a bounded additive disturbance on the observer's gain s.t. $\|\Delta(t)\| \leq r$ for all $t$.

The working hypotheses and lemmas used in this paper are the following: 
Assumption 1: System (10) is R-detectable and impulseobservable [18].

Assumption 2: All the nonlinear terms of the system (10) are Lipschitz, i.e. there exist $\alpha_{1}>0$ and $\alpha_{2}>0$ for which the functions $\gamma(t, x)$ and $\tilde{H}(x)$ satisfy:

$$
\begin{gathered}
\left\|\gamma\left(t, x_{1}\right)-\gamma\left(t, x_{2}\right)\right\| \leqslant \alpha_{1}\left\|x_{1}-x_{2}\right\|, \\
\left\|\tilde{H}\left(t, x_{1}\right)-\tilde{H}\left(t, x_{2}\right)\right\| \leqslant \alpha_{2}\left\|x_{1}-x_{2}\right\| .
\end{gathered}
$$

Assumption 3: The parameter vector $\hat{R}_{s c}$ is piecewise constant and bounded:

$$
\left\|\hat{R}_{s c}\right\| \leqslant \alpha_{3}, \quad \alpha_{3}>0
$$

Lemma 1 [19]

Let $\mathrm{x}$ and $\mathrm{y}$ be real vectors of the same dimension. Then $\forall \varepsilon>0$ the following inequality holds:

$$
2 x^{T} y \leqslant \varepsilon x^{T} x+\varepsilon^{-1} y^{T} y .
$$

\section{Lemma 2: Schur complement [20]}

Given any real matrices $X_{1}, X_{2}$ and $X_{3}$ s.t. $X_{1}=X_{1}^{T}$ and $X_{3}>0$, then

$$
X_{1}+X_{2}^{T} X_{3}^{-1} X_{2}<0
$$

if and only if

$$
\left[\begin{array}{cc}
X_{1} & X_{2}^{T} \\
X_{2} & -X_{3}
\end{array}\right]<0
$$

\section{Lemma 3: Barbalat's lemma [21]}

Let $\phi: R \rightarrow R$ be a uniformly continuous function on $[0,+\infty[$. Suppose that $\lim _{t \rightarrow+\infty} \int_{0}^{t} \phi(\tau) d \tau$ exists and is finite, then

$$
\lim _{t \rightarrow+\infty} \phi(t)=0 .
$$

The adaptive resilient observer error dynamics is given by:

$$
\begin{aligned}
E \dot{e}_{1} & =(A-K C-\Delta C) e_{1}+D_{1}(\gamma(t, x)-\gamma(t, \hat{x})) B_{m} \\
& +\left(\tilde{H}(x) R_{s c}-\tilde{H}(\hat{x}) \hat{R}_{s c}\right) .
\end{aligned}
$$

where $e_{1}=x-\hat{x}$ is the state estimation error.

Inspired by [14], sufficient conditions for the convergence of the nonlinear adaptive resilient observer error dynamics are provided by the following theorem.

Theorem Consider the following adaptation law:

$$
\dot{\hat{R}}_{s c}=\Sigma^{-1} \tilde{H}(\hat{x})^{T} \eta C(x-\hat{x})+\Sigma^{-1} L(G-\hat{G}) .
$$

where $L \in R^{-}$is a constant gain, $\Sigma^{-1}=\Sigma^{-T}>0$ an arbitrary constant matrix known as the adaptation gain and $\eta \in R^{q \times r}$ s.t. $P=\eta C$.

The system (11) is an asymptotically stable adaptive resilient observer for system (10), if there exist a non-singular matrix $P \in R^{n \times n}$, a matrix $W \in R^{r \times n}$ s.t. $K=\left(W P^{-1}\right)^{T}$ and scalars $\varepsilon_{1}>0, \varepsilon_{2}>0, \varepsilon_{3}>0$ for which the following matrix inequalities are solvable

$$
\begin{aligned}
& E^{T} P=P^{T} E \geq 0 \\
& {\left[\begin{array}{cccc}
\Lambda & P^{T} & P^{T} & P^{T} \\
P & -\varepsilon_{1} I & 0 & 0 \\
P & 0 & -\varepsilon_{2} I & 0 \\
P & 0 & 0 & -\varepsilon_{3} I
\end{array}\right]<0 .}
\end{aligned}
$$

where $\Lambda=A^{T} P+P^{T} A-C^{T} W-W^{T} C+\varepsilon_{1} \alpha_{1}^{2} B_{m}^{2}\left\|D_{1}\right\|^{2} I+$ $\varepsilon_{2} \alpha_{2}^{2} \alpha_{3}^{2} I+\varepsilon_{3} r^{2} C^{T} C$.

Furthermore, if the persistent excitation condition is verified $\forall t_{0}, \exists \xi, \delta>0$ s.t.

$$
\int_{t_{0}}^{t_{0}+\delta} \tilde{H}(\hat{x}) \tilde{H}(\hat{x})^{T} d \tau>\xi I,
$$

then, the vector of fouling thermal resistance estimates converges to its true value for all disturbances satisfying $\|\Delta(t)\| \leq r$.

Proof In order to prove the convergence of the nonlinear adaptive resilient observer (11) to the system (10), the proof of the asymptotic stability of the error dynamics is derived.

The parameter estimation error is defined by $e_{2}=R_{s c}-\hat{R}_{s c}$ and the following Lyapunov function:

$$
V(t)=e_{1}^{T} E^{T} P e_{1}+e_{2}^{T} \Sigma e_{2} .
$$

Deriving $V(t)$ with respect to time:

$$
\dot{V}(t)=\left(E \dot{e}_{1}\right)^{T} P e_{1}+e_{1}^{T} P^{T} E \dot{e}_{1}+2 e_{2}^{T} \Sigma \dot{e}_{2} .
$$

Let $\bar{A}=(A-K C-\Delta C)$. Then, equation (23) becomes

$$
\begin{aligned}
\dot{V}(t)= & {\left[\bar{A} e_{1}+D_{1}(\gamma(t, x)-\gamma(t, \hat{x})) B_{m}\right.} \\
& \left.+\left(\tilde{H}(x) R_{s c}-\tilde{H}(\hat{x}) \hat{R}_{s c}\right)\right]^{T} P e_{1} \\
& +e_{1}^{T} P^{T}\left[\bar{A} e_{1}+D_{1}(\gamma(t, x)-\gamma(t, \hat{x})) B_{m}\right. \\
& \left.+\left(\tilde{H}(x) R_{s c}-\tilde{H}(\hat{x}) \hat{R}_{s c}\right)\right]+2 e_{2}^{T} \sum \dot{e}_{2}, \\
= & e_{1}^{T}\left[\bar{A}^{T} P+P^{T} \bar{A}\right] e_{1}+2\left[D_{1}(\gamma(t, x)-\gamma(t, \hat{x})) B_{m}\right. \\
& \left.+\left(\tilde{H}(x) R_{s c}-\tilde{H}(\hat{x}) \hat{R}_{s c}\right)\right]^{T} P e_{1}+2 e_{2}^{T} \sum \dot{e}_{2} .
\end{aligned}
$$

Substituting $\hat{R}_{s c}=R_{s c}-e_{2}$ leads to:

$$
\begin{aligned}
\dot{V}(t)= & e_{1}^{T}\left[\bar{A}^{T} P+P^{T} \bar{A}\right] e_{1}+2\left[D_{1}(\gamma(t, x)-\gamma(t, \hat{x})) B_{m}\right]^{T} P e_{1} . \\
& +2\left[(\tilde{H}(x)-\tilde{H}(\hat{x})) R_{s c}\right]^{T} P e_{1} \\
& +2\left[\tilde{H}(\hat{x}) e_{2}\right]^{T} P e_{1}+2 e_{2}^{T} \Sigma \dot{e}_{2} .
\end{aligned}
$$

Applying Lemma 1 to (25) gives

$$
\begin{aligned}
\dot{V}(t) \leqslant & e_{1}^{T}\left[\bar{A}^{T} P+P^{T} \bar{A}\right] e_{1}+\varepsilon_{1}\left\|D_{1}(\gamma(t, x)-\gamma(t, \hat{x})) B_{m}\right\|^{2} \\
& +\varepsilon_{2}\left\|(\tilde{H}(x)-\tilde{H}(\hat{x})) R_{s c}\right\|^{2} \\
& +\left(\varepsilon_{1}^{-1}+\varepsilon_{2}^{-1}\right) e_{1}^{T} P^{T} P e_{1} \\
& +2\left[\tilde{H}(\hat{x}) e_{2}\right]^{T} P e_{1}+2 e_{2}^{T} \Sigma \dot{e}_{2} .
\end{aligned}
$$


Based on the assumptions on $\gamma(t, x), \tilde{H}(\hat{x}), R_{s c}$ and $\Delta$ ((12), (13), (14) and $\|\Delta(t)\| \leq r$, respectively), equation (26) becomes

$$
\begin{aligned}
\dot{V}(t) & \leqslant e_{1}^{T}\left[\Lambda+\left(\varepsilon_{1}^{-1}+\varepsilon_{2}^{-1}+\varepsilon_{3}^{-1}\right) P^{T} P\right] e_{1} \\
& +2\left[\tilde{H}(\hat{x}) e_{2}\right]^{T} P e_{1}+2 e_{2}^{T} \sum \dot{e}_{2} .
\end{aligned}
$$

where $\Lambda=\Omega+\varepsilon_{3} r^{2} C^{T} C$,

with $\Omega=A^{T} P+P^{T} A-C^{T} W-W^{T} C+\left(\varepsilon_{1} \alpha_{1}^{2} B_{m}^{2}\left\|D_{1}\right\|^{2}+\right.$ $\left.\varepsilon_{2} \alpha_{2}^{2} \alpha_{3}^{2}\right) I$.

Since $R_{s c}$ is piecewise constant, it results that $\dot{R}_{s c}=0$ and $\dot{e}_{2}=-\hat{R}_{s c}$. Based on this and substituting the adaptation law (19) yield to

$$
2\left[\tilde{H}(\hat{x}) e_{2}\right]^{T} P e_{1}+2 e_{2}^{T} \sum \dot{e}_{2}=-2 e_{2}^{T} L(G-\hat{G}) .
$$

The term $-e_{2}^{T} L(G-\hat{G})$ is always negative. In fact:

$$
\left\{\begin{array}{l}
\text { if } e_{2}<0 \rightarrow(G-\hat{G})>0, \text { then }-e_{2}^{T} L(G-\hat{G})<0 . \\
\text { if } e_{2}>0 \rightarrow(G-\hat{G})<0, \text { then }-e_{2}^{T} L(G-\hat{G})<0 .
\end{array}\right.
$$

Inequality (27) becomes

$$
\dot{V}(t) \leqslant e_{1}^{T}\left[\Lambda+\left(\varepsilon_{1}^{-1}+\varepsilon_{2}^{-1}+\varepsilon_{3}^{-1}\right) P^{T} P\right] e_{1} .
$$

It appears then that a sufficient condition for $\dot{V}(t)<0$ is

$$
\Lambda+\left(\varepsilon_{1}^{-1}+\varepsilon_{2}^{-1}+\varepsilon_{3}^{-1}\right) P^{T} P<0 .
$$

Inequality (31), which is nonlinear with respect to $\mathrm{P}$, can be expressed using Schur complement (Lemma 2) as a LMI.

$$
\left[\begin{array}{cccc}
\Lambda & P^{T} & P^{T} & P^{T} \\
P & -\varepsilon_{1} I & 0 & 0 \\
P & 0 & -\varepsilon_{2} I & 0 \\
P & 0 & 0 & -\varepsilon_{3} I
\end{array}\right]<0 .
$$

For certain $\beta>0$, inequality (31) can be written as follows

$$
\Lambda+\left(\varepsilon_{1}^{-1}+\varepsilon_{2}^{-1}+\varepsilon_{3}^{-1}\right) P^{T} P<-\beta I .
$$

Substituting (33) in (30):

$$
\dot{V}(t) \leqslant-\beta e_{1}^{T} e_{1} .
$$

Since $V(t)>0$ and $V(t)$ is strictly decreasing, $\mathrm{V}(\mathrm{t})$ is bounded. Therefore, $e_{1}$ and $e_{2}$ are bounded due to (22). Integrating (34) from $t=0$ to $t=t_{1}$ results in :

$$
V\left(t_{1}\right) \leqslant V(0)-\beta \int_{0}^{t_{1}} e_{1}^{T}(\tau) e_{1}(\tau) d \tau .
$$

Since $V(t)$ is bounded, we obtain from (35) that $e_{1} \in L^{2}$. Moreover, it can be concluded from the observer's error dynamic (18) that $\dot{e_{1}} \in L^{\infty}$.

Then by applying Barbalat's lemma (Lemma 3), we obtain

$$
\lim _{t \rightarrow \infty} e_{1}=0 \text { and } \lim _{t \rightarrow \infty} \dot{e}_{1}=0 .
$$

As a result, we obtain from (18)

$$
\lim _{t \rightarrow \infty} D_{1}(\gamma(t, x)-\gamma(t, \hat{x})) B_{m}+\left(\tilde{H}(x) R_{s c}-\tilde{H}(\hat{x}) \hat{R}_{s c}\right)=0 .
$$

As $\lim _{t \rightarrow \infty} \hat{x}=x$, (36) is reduced to

$$
\lim _{t \rightarrow \infty}\left(\tilde{H}(x)\left(R_{s c}-\hat{R}_{s c}\right)\right)=0 .
$$

Using the assumption of persistent excitation (21), the parameter estimation error converges to zero $\left(\lim _{t \rightarrow \infty} e_{2}=0\right)$, thus $\hat{R}_{s c} \rightarrow R_{s c}$.

\section{Simulation Results}

The estimation and localization performance of the proposed adaptive resilient observer is compared with the adaptive observer used in [13] with the same DCMD module. In order to reduce the computational cost and the execution time of the program, the module is divided into 3 cells and therefore, three fouling thermal resistances have to be estimated. Note that apart from computational limitations due to the LMIs resolution, the proposed observer works properly for a larger number of cells.

The following simulation is conducted: different gradual fouling resistances are imposed for the first, second and third cells starting from zero at $10 s, 20 s$ and $30 s$, respectively. Then, their values are stabilized at $80 \mathrm{~s}$ with respectively $2.5 \times 10^{-3}, 2 \times 10^{-3}, 1.5 \times 10^{-3}$ for 20 more seconds. The chosen resistance profile requires greater responsiveness from the observer which makes it challenging for the observer to give good performances. The simulation was performed with a constant input velocity of $0.39 \mathrm{~m} / \mathrm{s}$, feed and permeat inlet temperatures of $65^{\circ} \mathrm{C}$ and $20^{\circ} \mathrm{C}$, respectively.

Matrices $P$ and $W$ are computed by solving the LMIs using YALMIP toolbox [22] and the simulation of the proposed observer was conducted using the Matlab solver ode15s.

Fig. 3 shows the actual and estimated profiles of the fouling resistances $R_{S C}$ using the proposed observer. For comparison purposes, the estimated $R_{s c}$ obtained using the observer in [13] is also plotted. While the observer in [13] yields to a static error in $R_{s c}$ estimation, the obtained gain from the resilient observer design offers an accurate tracking of the fouling evolution in the three cells, despite the fact that Assumption 3 is not satisfied (i.e. $R_{s c} \neq 0$ ) which affects the observer's convergence (see therorem proof). However, this is no longer a problem since, thanks to the resilient term of the adaptive resilient observer, the bias introduced by $R_{s c}$ is compensated. Hence, the adaptive resilient observer presents better estimation and localization performance (i.e. it is able to identify which cell of the module is affected by fouling) than the classical one.

The simulated and estimated spacial distributions of the DCMD module feed and permeate inner temperatures are illustrated in Fig. 4 and Fig. 5, respectively. It is striking that the adaptive resilient observer provides a better temperature estimation than the adaptive observer in [13].

These numerical simulations show that the adaptive resilient observer is able to estimate simultaneously the module's inner temperatures and the fouling thermal resistance in the three cells with high accuracy. 

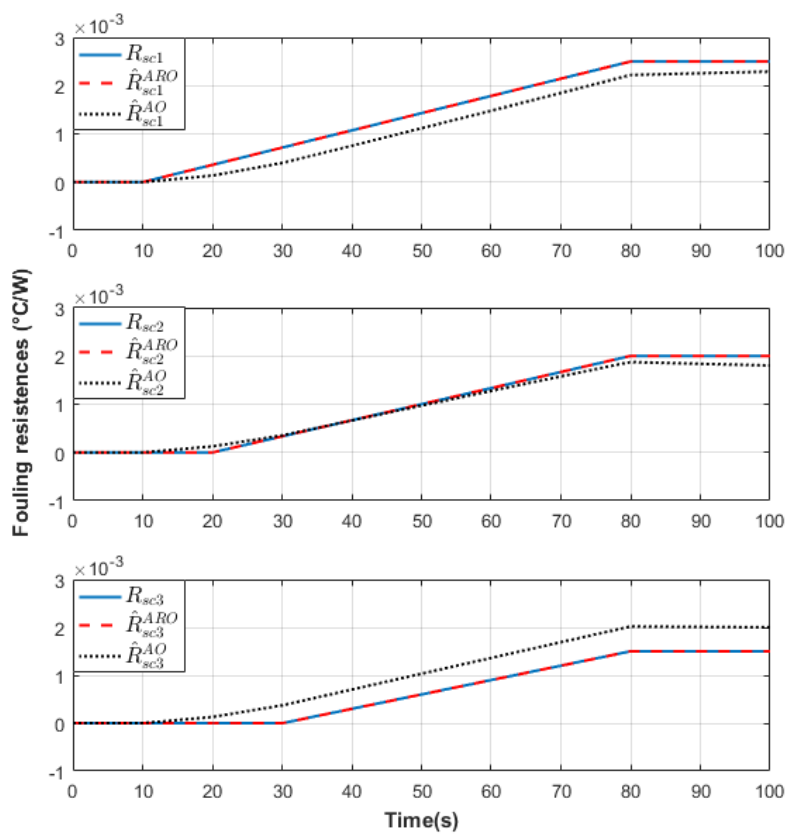

Fig. 3. Fouling resistances estimation with the adaptive resilient observer (ARO) vs. the adaptive observer (AO) of [13]

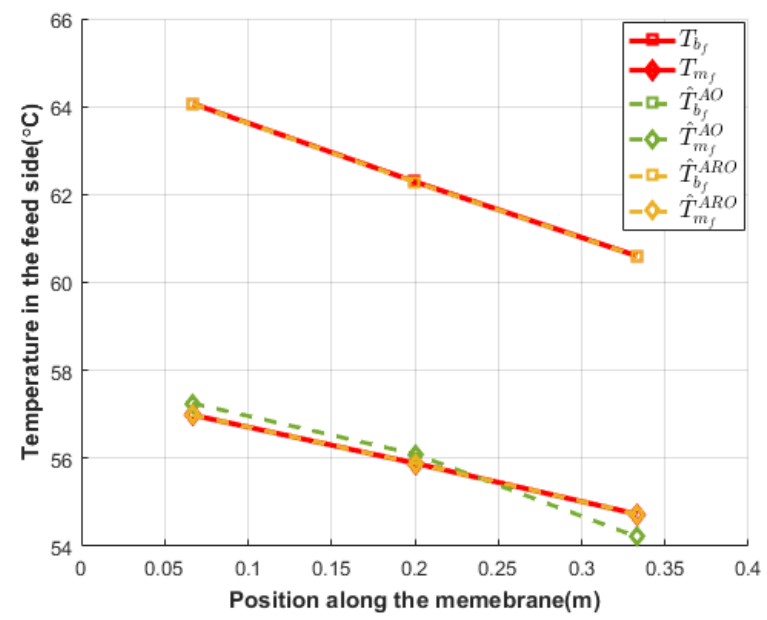

Fig. 4. Actual and estimated feed temperature distribution through the DCMD with the adaptive resilient observer (ARO) vs. the adaptive observer (AO) of [13] at $t=100 \mathrm{~s}$

\section{CONCLUSION}

This paper proposes a generalization of the results of fouling detection in DCMD systems to include fouling localization along the membrane. A nonlinear adaptive resilient observer is designed for that purpose and provides a simultaneous fouling thermal resistance and state estimation in the presence of a bounded disturbance on the gain's coefficient. Simulation results are shown in order to compare this approach to the classical adaptive observer presented in [13] and highlight the outperformance of the adaptive resilient observer in both fouling detection and localization

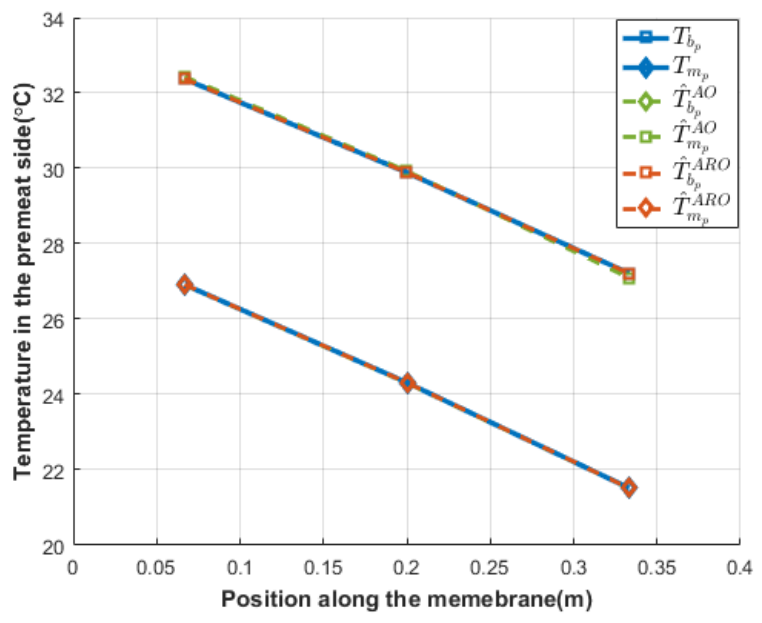

Fig. 5. Actual and estimated permeate temperature distribution through the DCMD with the adaptive resilient observer (ARO) vs. the adaptive observer (AO) of [13] at $t=100 \mathrm{~s}$

through a more accurate estimation of the fouling thermal resistance $R_{s c}$ as well as the states, thanks to the larger space of Lipschitz constants it offers for solving the LMIs. Finally, future work will focus on the experimental validation of the proposed fouling detection method.

\section{ACKNOWLEDGMENT}

This work has been supported by the King Abdullah University of Science and Technology (KAUST) Base Research Fund (BAS/1/1627-01-01) to Taous Meriem Laleg.

\section{REFERENCES}

[1] G. Micale, A. Cipollina, and L. Rizzuti, "Seawater Desalination for Freshwater Production" in Seawater Desalination, Eds. Berlin, Heidelberg: Springer-Verlag, 2009, pp. 1-15.

[2] Z. S. Tai, M. Aziz, M. H. Othman, A. Ismail, M. Rahman, J. Jaafar, "An Overview of Membrane Distillation" in Membrane Separation Principles and Applications, Elsevier, 2019, pp.251-281

[3] M. Khayet and T. Matsuura, Membrane distillation principles and applications, Elsevier, 2011.

[4] M. Gryta, "Fouling in direct contact membrane distillation process," Journal of Membrane Science, Vol. 325, pp. 383-394, Aug 2008.

[5] K. Scott, Handbook of Industrial Membranes, Kidlington, UK: Elsevier, 1997.

[6] A.I. Schäfer, A.G. Fane, and T.D. Wait (Eds.), Nanofiltration: Principles and Application, Oxford: Elsevier, 2005.

[7] M. Al-Shammiri, M. Safar, and M. Al-Dawas, "Evaluation of two different antiscalants in real operation at the Doha research plant," Desalination, vol. 128, pp. 1-16, 2000.

[8] G. -Y. Chai, A. R. Greenberg, and W. B. Krantz, "Ultrasound, gravimetric, and SEM studies of inorganic fouling in spiral-wound membrane modules," Desalination, vol. 208, no. 1-3, pp. 277-293, 2007.

[9] L. N. Sim, Z. J. Wang, J. Gu, H. G. L. Coster, and A. G. Fane, "Detection of reverse osmosis membrane fouling with silica, bovine serum albumin and their mixture using in-situ electrical impedance spectroscopy," Journal of Membrane Science, vol. 443, pp. 45-53, 2013.

[10] H. Monclús, G. Ferrero, G. Buttiglieri, J. Comas, and I. RodríguezRoda, "Online monitoring of membrane fouling in submerged MBRs," Desalination, vol. 277, pp. 414-419, 2011.

[11] P. Marel, A. Zwijnenburg, A. Kemperman, M. Wessling, H. Temmink, and W. Meer, "An improved flux-step method to determine the critical flux and the critical flux for irreversibility in a membrane bioreactor," Journal of Membrane Science, vol. 332, pp. 24-29, Jan 2009. 
[12] F. Delmotte, M. Dambrine, S. Delrot, and S. Lalot, "Fouling detection in a heat exchanger: A polynomial fuzzy observer approach," Control Engineering Practice, vol. 21, no. 10, pp. 1386-1395, 2013.

[13] A. M. Karam and T. M. Laleg-Kirati, "Membrane fouling modeling and detection in direct contact membrane distillation," Journal of Process Control, vol. 81, pp. 190-196, 10.1016, 2019.

[14] M. Pourgholi and V. Majd, "A nonlinear adaptive resilient observer design for a class of lipschitz systems using LMI," Circuits, Systems, and Signal Processing, vol. 30, pp. 1401-1415, 2011.

[15] Chung Seop Jeong, E. E. Yaz, A. Bahakeem and Y. I. Yaz, "Resilient design of observers with general criteria using LMIs," 2006 American Control Conference, Minneapolis, MN, 2006, pp. 6.

[16] F. Wang and V. V. Tarabara, "Pore blocking mechanisms during early stages of membrane fouling by colloids," Journal of Colloid and Interface Science, vol. 328, no. 2, pp. 464-469, 2008.

[17] A. M. Karam and T. M. Laleg-Kirati, "Electrical thermal network for direct contact membrane distillation modeling and analysis," 2014 IEEE Conference on Control Applications, CCA, 2014, pp. 1563-1569.

[18] L. Dai, Singular Control Systems, Springer-Verlag, 1989.

[19] F. Chen and W. Zhang, "LMI criteria for robust chaos synchronization of a class of chaotic systems," Nonlinear Analysis: Theory, Methods and Applications, vol. 67, no. 12, pp. 3384 - 3393, 2007.

[20] J. Gallier, Notes on the Schur Complement, 2010.

[21] H. K. Khalil, Nonlinear Systems, 3rd ed., Prentice Hall, 2002.

[22] J. Lofberg, "YALMIP : a toolbox for modeling and optimization in MATLAB," 2004 IEEE International Conference on Robotics and Automation, New Orleans, LA, 2004, pp. 284-289. 